\title{
Correction to: Acute Exacerbation of Chronic Hepatitis B
}

\author{
Qin Ning
}

\section{Correction to: \\ Q. Ning (ed.), Acute Exacerbation of Chronic Hepatitis B, https://doi.org/10.1007/978-94-024-1606-0}

The original version of the book was inadvertently published with spelling errors in author names. The names have been corrected and details of the changes have been provided below.

Acknowledgements: Dong Xu has been changed to Dong Xi.

Contents (Chapter 2): Dong Xu has been changed to Dong Xi.

Contents (Chapter 3): Yi-Ming Zhang has been changed to Ji-Ming Zhang.

Advisory Board (in alphabetical order): Dong Xu has been changed to Dong Xi, Hai-Bing Su has been changed to Hai-Bin Su, Yi-Ming Zhang has been changed to Ji-Ming Zhang, Jin-Ming Zhao has been changed to Jing-Ming Zhao.

In page 49, Dong Xu has been changed to Dong Xi.

In page 119, Yi-Ming Zhang has been changed to Ji-Ming Zhang.

The updated online version of these chapters can be found at https://doi.org/10.1007/978-94-024-1606-0_2

https://doi.org/10.1007/978-94-024-1606-0_3 